\title{
An Optimal Economic Dispatch Algorithm for Large Scale Power Systems with Cogeneration Units
}

\author{
Chao-Lung Chiang
}

\begin{abstract}
This paper proposes an optimal economic dispatch algorithm for large scale power systems with cogeneration units. A hybrid differential evolution with multiplier updating (HDE-MU) is introduced to solve the combined heat and power economic dispatch (CHPED) problems. The hybrid differential evolution (HDE) has the ability to efficiently search and actively explore solutions. Multiplier updating (MU) is introduced to avoid deforming the augmented Lagrange function (ALF), which is adopted to manage system constraints of the CHPED problem. The proposed HDE-MU integrates the HDE with the MU. A practical CHPED system with two cases of a basic case and a large case is employed to demonstrate that the proposed algorithm has the better performance in comparison with the previous methods, and the proposed HDE-MU has the effectiveness when applied to the large scale CHPED operation of power systems.
\end{abstract}

Index Terms-Cogeneration, differential evolution, economic dispatch, multiplier updating, large scale.

\section{INTRODUCTION}

The conversion of fossil fuel into electricity is not an efficient process. Even the energy efficiency of the most modern combined cycle plants are between 50 and $60 \%$ efficient [1]. Most of the energy wasted in the conversion process is heat. Recently, combined heat and power (CHP) units have played an increasingly important role in the utility industry [2 7]. Complexity arises if one or more units produce both power and heat. Such a case, both the power demand and the heat demand must be satisfied. Cogeneration units provide both electrical power and heat to customers. The heat production capacity of most cogeneration units, depend on the power generated, and vice versa. The mutual dependency of multiple-demand and Heat-Power capacity of those units introduce complexities in the integration of cogeneration units into the economic dispatch problem. Solving such a complex optimization problem requires powerful techniques. Non-linear optimal algorithms, such as dual and quadratic programming [8], and gradient descent approaches, such as Lagrangian relaxation [9], have been applied for solving CHPED problems. However, these methods cannot handle nonsmooth non-convex fuel cost function of the conventional thermal generator. The advent of stochastic search algorithms has overcome this problem for solving CHPED problems. Group search optimization [1], cuckoo search algorithm [2], real coded genetic algorithm with improved

Manuscript published November 16, 2016.

Chao-Lung Chiang, Department of Electronic Engineering, Nan Kai University of Technology, Nan-Tou 542, Taiwan, ROC. (e-mail: t129@nkut.edu.tw).
Mühlenbein mutation [3], crisscross optimization algorithm [4], oppositional teaching learning based optimization [5], hybrid harmony search with arithmetic crossover operation [6], improved group search optimization [7], Generalized algebraic modeling system (GAMS) [10], canonical coordinates method (CCM) [11], harmony search with genetic algorithm (HSGA) [12], harmony search for economic dispatch (EDHS) [13], cuckoo optimization algorithm with penalty function (PFCOA) [14], gravitational search algorithm (GSA) [15], exchange market algorithm (EMA) [16], benders decomposition (BD) [17], particle swarm optimization with time varying acceleration coefficients (TVAC-PSO) [18], firefly algorithm (FA) [19], invasive weed optimization (IWO) [20], and harmony search (HS) [21] have been applied to solve the CHPED problems. The results obtained by the proposed algorithm, involving $\mathrm{HDE}$ and $\mathrm{MU}$, have been compared with the existing methodologies reported in recent literature.

Storn and Price [22] developed the Differential evolution (DE) which immediately gained popularity as a robust evolutionary algorithm. DE has been widely applied to the optimization problems in the power systems [23 27]. Throughout the years, DE has been used extensively for optimization problems, many results of which are the best compared to other standard methodologies. However, it has problems of converging onto local optimal solutions. A migration [28] is embedded in the proposed HDE to overcome such drawbacks for solving CHPED problems. The migration operation is included in the HDE to regenerate a newly diverse population, which prevents individuals from gradually clustering and thus greatly increases the amount of search space explored for a small population.

The reminder of this paper is organized as follows: Section II introduces the mathematical formulation of the CHPED problem. Section III describes the proposed algorithm involving HDE and MU. Section IV presents two cases of one basic case and one large scale case for testing the CHPED problem, compares the results obtained by HDE-MU with other methods reported in the literature. Conclusions are finally in Section V.

\section{FORMULATION OF THE CHPED PROBLEM}

\section{A. Objective function}

The CHPED problem is to determine the unit power and heat production so that the system's production cost is minimized while the power and heat demands and other constraints are met. It can be mathematically stated as, 
Minimize

$$
\sum_{i=1}^{n_{p}} \operatorname{Cost}_{i}\left(P_{i}\right)+\sum_{j=1}^{n_{b}} \operatorname{Cost}_{j}\left(H_{j}, P_{j}\right)+\sum_{k=1}^{n_{h}} \operatorname{Cost}_{k}\left(H_{k}\right)
$$

Where

$$
\begin{gathered}
\operatorname{Cost}_{i}\left(P_{i}\right)=a_{i}+b_{i} P_{i}+c_{i} P_{i}^{2}+d_{i} P_{i}^{3} \\
\operatorname{Cost}_{j}\left(H_{j}, P_{j}\right)=a_{j}+b_{j} P_{j}+c_{j} P_{j}^{2}+d_{j} H_{j}^{2}+r_{j} H_{j}+w_{j} P_{j} H_{j}(3) \\
\operatorname{Cost}_{k}\left(H_{k}\right)=a_{k}+b_{k} H_{k}+c_{k} H_{k}^{2}
\end{gathered}
$$

and where $P$ stands unit power generation; $H$ is unit heat production; $\operatorname{Cost}_{i}\left(P_{i}\right)$ with $i=1,2, \ldots, n_{p}$ represents the cost function of the $i^{\text {th }}$ power-only unit in $\$ / \mathrm{h}$ and $a_{i}, b_{i}, c_{i}$, and $d_{i}$ are cost coefficients of generator $i$; $\operatorname{Cost}_{j}\left(H_{j}, P_{j}\right)$ with $j=1,2, \ldots, n_{b}$ is the fuel cost function of the $j^{\text {th }}$ CHP unit in $\$ / \mathrm{h}$ and $a_{j}, b_{j}, c_{j}, d_{j}, r_{j}$, and $w_{j}$ are cost coefficients of unit $j$; $\operatorname{Cost}_{k}\left(H_{k}\right)$ with $k=1,2, \ldots, n_{h}$ represents the cost function of the $k^{\text {th }}$ heat-only unit in $\$ / \mathrm{h}$ and $a_{k}, b_{k}$, and $c_{k}$ are cost coefficients; $n_{p}, n_{b}$, and $n_{h}$ denote the number of power-only units, co-generation units, and heat-only units, respectively; subscripts of $i, j$, and $k$ are used for above mentioned unit; it should be mentioned that $\left(P_{i}, P_{j}\right)$ and $\left(H_{j}, H_{k}\right)$ are the output active power (in MW) and heat production (in MWth), respectively.

\section{B. Constraints}

Subject to the equilibrium constraints of electricity and heat production, and the capacity limits of each unit. Power and heat balance equations are represented by equality constraints (5) and (6), respectively.

$$
\begin{gathered}
\sum_{i=1}^{n_{p}} P_{i}+\sum_{j=1}^{n_{b}} P_{j}=P_{d} \\
\sum_{j=1}^{n_{b}} H_{j}+\sum_{k=1}^{n_{k}} H_{k}=H_{d} \\
P_{i}^{\min } \leq P_{i} \leq P_{i}^{\max }, \quad i=1, \cdots, n_{p} \\
P_{j}^{\text {min }}\left(H_{j}\right) \leq P_{j} \leq P_{j}^{\max }\left(H_{j}\right), \quad j=1, \cdots, n_{b} \\
H_{j}^{\text {min }}\left(P_{j}\right) \leq H_{j} \leq H_{j}^{\max }\left(P_{j}\right), \quad j=1, \cdots, n_{b} \\
H_{k}^{\min } \leq H_{k} \leq H_{k}^{\max }, \quad k=1, \cdots, n_{h}
\end{gathered}
$$

Where $P_{d}$ and $H_{d}$ are system power and heat demands, respectively. $P^{\min }$ and $P^{\max }$ are unit power capacity limits; $h^{\min }$ and $h^{\max }$ are unit heat capacity limits.

The CHPED problem clearly introduces the complication of more constraints than in required pure power economic dispatch problem. The insufficiencies difficulties with conventional methods thus follow from the fact that CHPED is a highly constrained optimization problem.

\section{THE PROPOSED HDE-MU}

\section{A. $D E$}

The DE algorithm is one of the population-based optimization algorithms. The steps for implementing DE are as follows [22]:

Step 1: Initial population: A population of $N_{P}$ initial solutions randomly distributed in the $n_{c}$ dimensional search space of the optimization problem, are initiated. The DE uses $N_{p}$ vectors of variables $x$ in the optimization problem, namely, $x^{G}=\left\{x_{i}^{G}, i=1, \ldots, N_{p}\right\}$, as a population in generation $G$. For convenience, the decision vector, $x_{i}$, is represented as $\left(x_{1 i} \ldots x_{j i} \ldots x_{n_{C} i}\right)$. Here, the decision variable, $x_{j i}$ is directly coded as a real value within its bounds of $\left(x_{j}^{\min }, x_{j}^{\max }\right)$. Each individual is generated as follows:

$$
\begin{gathered}
\left.x_{j i}^{G}\right|_{G=0}=x_{j}^{\min }+\operatorname{rand}(0,1) *\left(x_{j}^{\max }-x_{j}^{\min }\right) \\
j=1,2, \ldots, n_{c}, \quad i=1,2, \ldots, N_{P}
\end{gathered}
$$

Where $\operatorname{rand}(0,1)$ is a random number between 0 and 1 .

Step 2: Mutation operator: In mutation step, for each individual $x_{i}$ (target vector) of the new population, three different individuals $x_{r 1}, x_{r 2}$, and $x_{r 3}(r 1 \neq r 2 \neq r 3 \neq i)$ are pseudo-randomly extracted from the population to generate a new vector as:

$$
z_{j i}=x_{j r 1}+F^{*}\left(x_{j r 2}-x_{j r 3}\right) \quad j=1,2, \ldots, n_{c}
$$

Where $F \in[0,2]$ is a uniformly distributed random number which controls the length of the population exploration vector $\left(x_{r 2}-x_{r 3}\right)$.

Step 3: Crossover operator: After mutation step, the crossover operator, according to the following equation, is applied on the mutation vector $Z_{j i}$ and the vector $x_{j i}$ to generate the trial vector $U_{j i}$, for increasing population diversity of the mutation vector.

$$
\begin{aligned}
U_{j i} & = \begin{cases}z_{j i}, & \text { if rand }(0,1) \leq C R \\
x_{j i}, & \text { otherwise }\end{cases} \\
j & =1,2, \ldots, n_{c}, \quad i=1,2, \ldots, N_{P}
\end{aligned}
$$

Where $C R \in[0,1]$ is known as the crossover rate which is a constant.

Step 4: Selection \& evaluation operator: The selection \& evaluation process is repeated for each pair of target/trial vectors using the evaluation function $F\left(U_{j i}\right)$ to compare with the evaluation function value $F\left(x_{j i}\right)$, and the better one will be selected to be a member of the DE population generation for the next iteration $\left(x_{j i}^{G+1}\right)$.

\section{B. $H D E$}

In HDE [23 27], the one-to-one competition will have a faster convergence speed to give a higher probability toward a global optimum with much less computation time. It can use a small population in the evolutionary process to obtain a global solution. Generally, Evolutionary optimization 
involves two critical issues evolutionary direction and population diversity. As the evolutionary direction is effective in searching, the strong evolutionary direction can reduce the computational burden and increase the probability of rapidly finding an (possibly local) optimum. As population diversity is increased, the genotype of the offspring differs more from the parent. Accordingly, a highly diverse population can increase the probability of exploring the global optimum and prevent a premature convergence to a local optimum. These two important factors are here balanced by employing the migration [28] into HDE that can determine an efficacious direction in which to search for a solution and simultaneously maintain an appropriate diversity for a small population. The migrant individuals are generated based on the best individual, $x_{b}^{G+1}=\left(x_{1 b}^{G+1}, x_{2 b}^{G+1}, \ldots, x_{n_{C} b}^{G+1}\right)$, by non-uniformly random choice. Genes of the $i^{\text {th }}$ individual are regenerated according to

$$
x_{j i}^{G+1}=\left\{\begin{array}{ccc}
x_{j b}^{G+1}+\rho\left(x_{j}^{\min }-x_{j b}^{G+1}\right), & \text { if } & r_{1}<\frac{x_{j b}^{G+1}-x_{j}^{\min }}{x_{j}^{\max }-x_{j}^{\min }} \\
x_{j b}^{G+1}+\rho\left(x_{j}^{\max }-x_{j b}^{G+1}\right) & , \text { otherwise }
\end{array}\right.
$$

where $j=1, \cdots, n_{C} ; i=1, \cdots, N_{p}, r_{1}$ and $\rho$ are random numbers in the range of $[0,1]$. The migration may be performed if only the best fitness has not been improved for over 500 generations running, and the migrant population will not only become a set of newly promising solutions but also easily escape the local extreme value trap. More details of the HDE have shown in [23 27].

\section{C. $M U$}

Michalewicz et al. [29] surveyed and compared several constraint-handling techniques used in evolutionary algorithms. Among these techniques, the penalty function method is one of the most popularly used to handle constraints. In this method, the objective function includes a penalty function that is composed of the squared or absolute constraint violation terms. Powell [30] noted that classical optimization methods include a penalty function have certain weaknesses that become most serious when penalty parameters are large. More importantly, large penalty parameters ill condition the penalty function so that obtaining a good solution is difficult. However, if the penalty parameters are too small, the constraint violation does not contribute a high cost to the penalty function. Accordingly, choosing appropriate penalty parameters is not trivial. Herein, the MU [31] is introduced to handle this constrained optimization problem. Such a technique can overcome the ill conditioned property of the objective function. Considering the nonlinear problem with general constraints as follows: Where $h_{k}(x)$ and $g_{k}(x)$ stand for equality and inequality constraints, respectively.

$$
\begin{array}{lll} 
& \min _{x} f(x) \\
\text { subject to } & h_{k}(x)=0, \quad k=1, \ldots, m_{e} \\
& g_{k}(x) \leq 0, \quad k=1, \ldots, m_{i}
\end{array}
$$

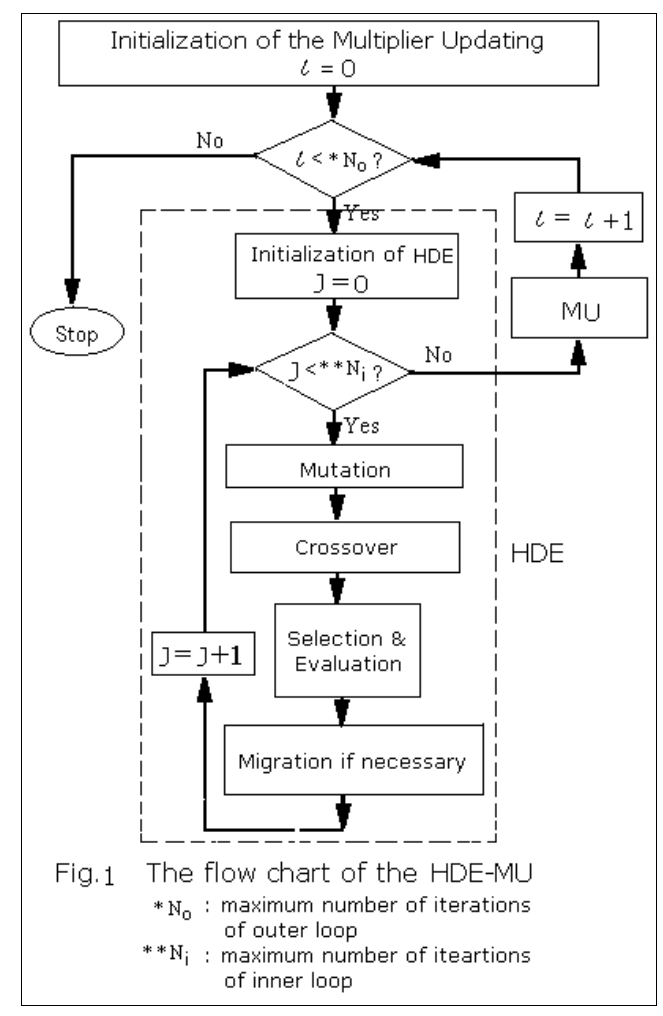

The augmented Lagrange function (ALF) [30] for constrained optimization problems is defined as:

$$
\begin{aligned}
L_{a}(x, v, v)= & f(x)+\sum_{k=1}^{m_{e}} \alpha_{k}\left\{\left[h_{k}(x)+v_{k}\right]^{2}-v_{k}^{2}\right\} \\
& +\sum_{k=1}^{m_{i}} \beta_{k}\left\{\left\langle g_{k}(x)+v_{k}\right\rangle_{+}^{2}-v_{k}^{2}\right\}
\end{aligned}
$$

where $\alpha_{k}$ and $\beta_{k}$ are the positive penalty parameters, and the corresponding Lagrange multipliers $v=\left(v_{1}, \ldots, v_{m_{e}}\right)$ and $v=\left(v_{1}, \ldots, v_{m_{i}}\right) \geq 0$ are associated with equality and inequality constraints, respectively.

The contour of the ALF does not change shape between generations while constraints are linear. Therefore, the contour of the ALF is simply shifted or biased in relation to the original objective function, $f(x)$. Consequently, small penalty parameters can be used in the MU. However, the shape of contour of $L_{a}$ is changed by penalty parameters while the constraints are nonlinear, demonstrating that large penalty parameters still create computational difficulties. Adaptive penalty parameters of the MU are employed to alleviate the above difficulties. More details of the MU have shown in [31].

\section{The proposed HDE-MU}

Figure 1 displays the flow chart of the proposed algorithm, which has two iterative loops. The ALF is used to obtain a minimum value in the inner loop with the given penalty parameters and multipliers, which are then updated in the outer loop toward producing an upper limit of $L_{a}$. When both inner and outer iterations become sufficiently large, the ALF converges to a saddle-point of the dual problem [29]. Advantages of the proposed HDE-MU are that the HDE efficiently searches the optimal solution in the economic dispatch process and the MU effectively tackles 
system constraints.

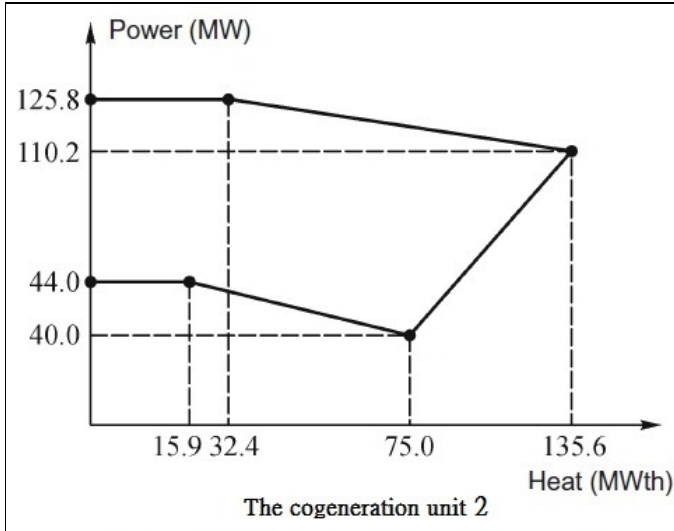

Fig. 2 Heat-power feasible operation region

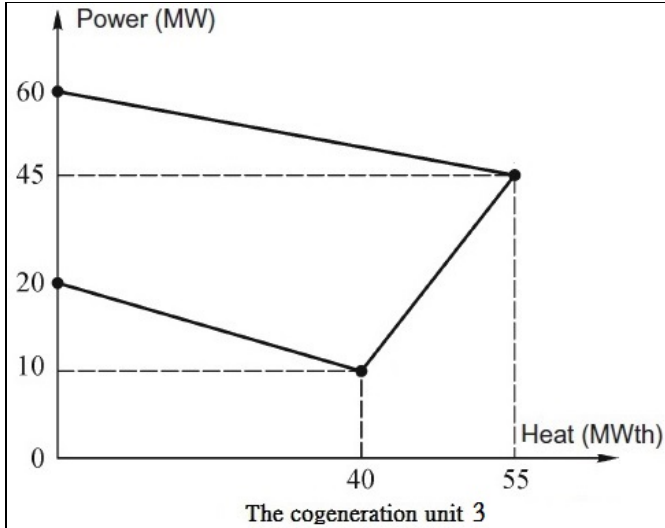

Fig. 3 Heat-power feasible operation region

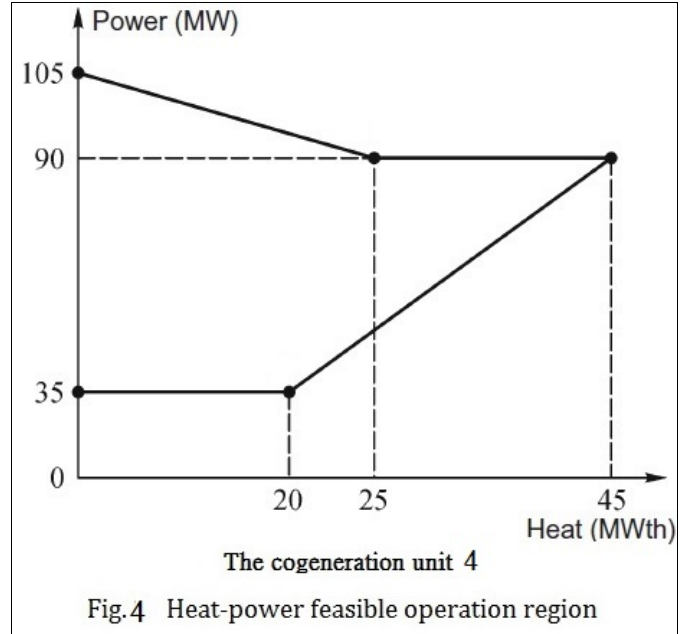

IV. SYSTEM SIMULATIONS

In this section, the proposed method is tested on two cases of one basic case and one large scale case for solving the CHPED problem. The propose approach solves CHPED problems considering capacity limits and heat-power mutural dependency constraints. The MU algorithm was used in HDE to hand the equality and inequality constraints. The computation was implemented on a personal computer (Intel(R) Core(TM) i7-3770 CPU @ 3.4 GHz with 8G Ram) in FORTRAN-90 language. Setting factors used in this two cases are as follows; the population size $N_{p}$ is set as 10 for HDE-MU. The iteration numbers of outer loop and inner loop are set to (outer, inner) as $(30,5000)$.

\section{A. The basic case}

A realistic CHPED system involvess one conventional power unit, three cogeneration units, and one heat-only unit, are employed to be the basic case for comparing the proposed HDE-MU with previous methods in qualities and performances of the optimal solutions obtained. The system power demand $\left(P_{d}\right)$ and heat demand $\left(H_{d}\right)$ of this CHPED problem are $300 \mathrm{MW}$ and $150 \mathrm{MW}$ th, respectively. This case used the linear boundary of the Heat-Power feasible region for cogeneration units. The associated data of this CHPED problem are the same as [21].

The feasible power and heat output region of cogeneration unit 2, 3 and 4 are shown in Figs 2, 3 and 4, respectively. These Figs 2, 3 and 4 demonstrate correlations between the power and heat outputs.

The implementation of the proposed algorithm for this basic case can be described as follows:

$$
\begin{aligned}
L_{a}(x, v, v) & =f(x)+\sum_{k=1}^{2} \alpha_{k}\left\{\left[h_{k}(x)+v_{k}\right]^{2}-v_{k}^{2}\right\} \\
& +\sum_{k=1}^{8} \beta_{k}\left\{\left\langle g_{k}(x)+v_{k}\right\rangle_{+}^{2}-v_{k}^{2}\right\}
\end{aligned}
$$

$$
\begin{aligned}
\text { objective: } & \min _{x=\left(P_{1}, P_{2}, P_{3}, P_{4}, H_{2}, H_{3}, H_{4}, H_{5}\right)} f(x) \\
f(x) & =\operatorname{Cost}_{1}\left(P_{1}\right)+\sum_{j=2}^{4} \operatorname{Cost}_{j}\left(H_{j}, P_{j}\right)+\operatorname{Cost}_{5}\left(H_{5}\right)
\end{aligned}
$$

where

$$
\begin{gathered}
\operatorname{Cost}_{1}\left(P_{1}\right)=0.000115 P_{1}^{3}+0.00172 P_{1}^{2}+7.6997 P_{1}+254.8863 \\
\operatorname{Cost}_{2}\left(H_{2}, P_{2}\right)=0.00435 P_{2}^{2}+36 P_{2}+0.027 H_{2}^{2}+ \\
0.6 H_{2}+0.011 P_{2} H_{2}+1250 \\
\operatorname{Cost}_{3}\left(H_{3}, P_{3}\right)=0.1035 P_{3}^{2}+34.5 P_{3}+0.025 H_{3}^{2}+ \\
2.203 H_{3}+0.051 P_{3} H_{3}+2650 \\
\operatorname{Cost}_{4}\left(H_{4}, P_{4}\right)=0.072 P_{4}^{2}+20 P_{4}+0.02 H_{4}^{2}+ \\
2.3 H_{4}+0.04 P_{4} H+1565
\end{gathered}
$$$$
\operatorname{Cost}_{5}\left(H_{5}\right)=0.038 H_{5}^{2}+2.0109 H_{5}+950
$$

Subject to;

$$
\begin{aligned}
& h_{1}: \quad P_{1}+P_{2}+P_{3}+P_{4}-P_{d}=0 \\
& h_{2}: \quad H_{2}+H_{3}+H_{4}+H_{5}-H_{d}=0 \\
& g_{1}: \quad 1.15841584158 H_{2}-P_{2}-46.8811881188 \leq 0 \\
& g_{2}:\left\{\begin{array}{c}
-P_{2}+44.0 \leq 0, \quad \text { if } H_{2}<15.9 \\
-0.0676818959 H_{2}-P_{2}+45.0761421319 \leq 0, \quad \text { if } H_{2} \geq 15.9
\end{array}\right. \\
& g_{3}:\left\{\begin{array}{c}
P_{2}-125.8 \leq 0, \quad \text { if } H_{2}<32.4 \\
0.1511627909 H_{2}+P_{2}-130.697674418 \leq 0, \quad \text { if } \quad H_{2} \geq 32.4
\end{array}\right. \\
& g_{4}: \quad-0.25 H_{3}-P_{3}+20 \leq 0 \\
& g_{5}: \quad \begin{array}{l}
7 H_{3}-3 P_{3}-250 \leq 0 \\
g_{6}: \\
3 H_{3}+11 P_{3}-660 \leq 0
\end{array} \\
& g_{7}:\left\{\begin{array}{c}
-P_{4}+35.0 \leq 0, \quad \text { if } \quad H_{4}<20.0 \\
2.2 H_{4}-P_{2}-9 \leq 0, \quad \text { if } \quad H_{4} \geq 20.0
\end{array}\right. \\
& g_{8}:\left\{\begin{array}{c}
P_{4}-90.0 \leq 0, \quad \text { if } \quad H_{4}>25.0 \\
2.4 H_{4}+P_{2}-150 \leq 0, \quad \text { if } \quad H_{4} \leq 25.0
\end{array}\right.
\end{aligned}
$$


TABLE1: COMPARES FIFTEEN COMPUTATIONAL RESULTS OBTAINED FROM THE PROPOSED HDE-MU AND PREVIOUS METHODS.

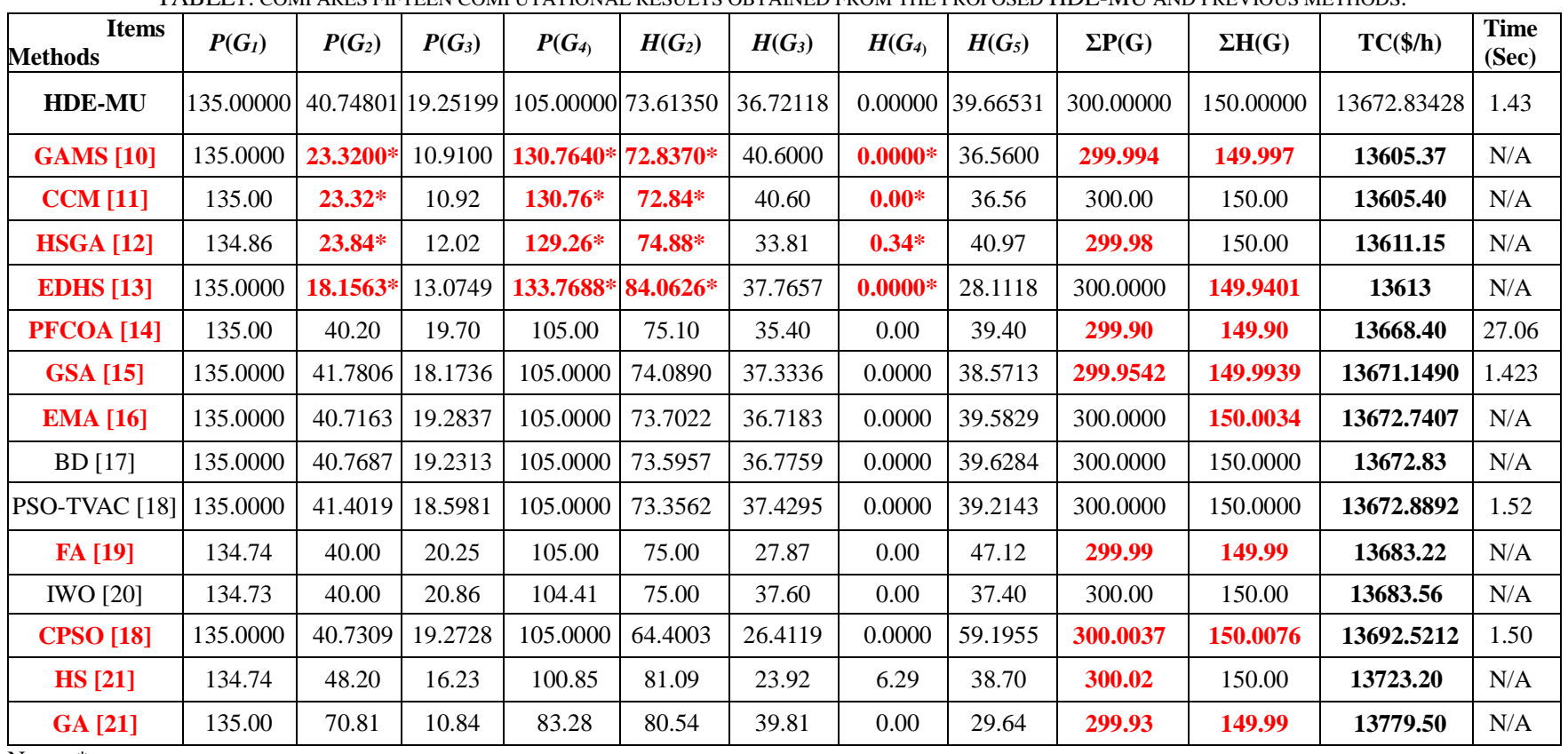

NOTE: * DENOTING OPERATION IN THE INFEASIBLE REGION.

This minimum cost problem consists of one objective function with eight variable parameters, $\left(P_{1}, P_{2}, P_{3}, P_{4}, H_{2}\right.$, $H_{3}, H_{4}$, and $H_{5}$ ), two equality constraints, $\left(h_{1}\right.$ and $h_{2}$ ), and eight inequality constraints, $\left(g_{1} \sim g_{8}\right)$, in the Heat-Power available region.

This basic case shows the validly and practicability of general use for the CHPED problems. Table 1 compares fifteen computational results obtained from the proposed HDE-MU, generalized algebraic modeling system (GAMS) [10], canonical coordinates method (CCM) [11], harmony search with genetic algorithm (HSGA) [12], harmony search for economic dispatch (EDHS) [13], cuckoo optimization algorithm with penalty function (PFCOA) [14], gravitational search algorithm (GSA) [15], exchange market algorithm (EMA) [16], benders decomposition (BD) [17], particle swarm optimization with time varying acceleration coefficients (TVAC-PSO) [18], firefly algorithm (FA) [19], invasive weed optimization (IWO) [20], classic PSO (CPSO) [19], harmony search (HS) [21] and genetic algorithm (GA) [21]. Although results obtained from GAMS [10], CCM [11], HSGA [12] and EDHS [13] have the less cost than the proposed HDE-MU, the bookmarks indicate that their outputs of $\left(\mathrm{H}_{2}, \mathrm{P}_{2}\right)$ and $\left(\mathrm{H}_{4}\right.$, $\mathrm{P}_{4}$ ) are invade infeasible heat-power regions. Hence, the following works are not considered competitive: GAMS [10], CCM [11], HSGA [12] and EDHS [13]. The heat outputs and/or power outputs of PFCOA [14], GSA [15], EMA [16], FA [19], IWO [20], CPSO [18], HS [21] and GA [21] didn't match the heat demand ( $\left.H_{d}=150 \mathrm{MWth}\right)$ and power demand $\left(P_{d}=300 \mathrm{MW}\right)$ that will cause an unbalance of system's heat and power demands. By investigating the results presented in Table 1, it is observed that the best total cost utilizing HDE-MU is $13,672.83428$ $\$ / h$, which is much less than the best results previously reported in PSO-TVAC [18] and IWO [20], and very close to the best result of $\mathrm{BD}$ [17]. As seen from the best solution of HDE-MU listed in Table 1, the power output is 300.00000 MW and the heat output is 150.00000 MWth.

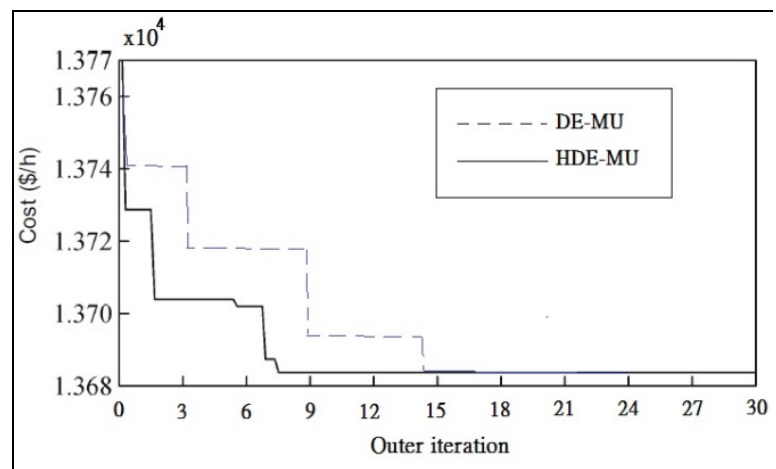

Fig.5 Convergence characteristics of the proposed HDE-MU in comparison with DE-MU.

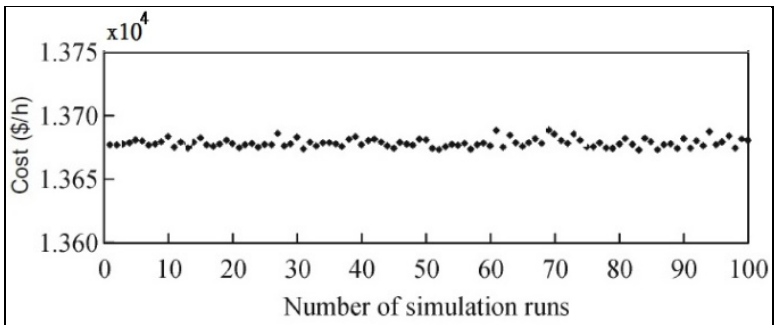

Fig.6 Distribution of the minimum costs in 100 trials

Apparently, the equality constraints (5) and (6) of power and heat demands are fully satisfied. Therefore, the result obtained from the proposed HDE-MU is an optimal and feasible solution. According to the absolute CPU time of different methods listed in the Table 1, the proposed HDEMU is faster than most of the compared algorithms, but slightly slower than GSA [15]. Consequently, the HDEMU is not only supplying the exact total of heat and power, but also has the less CPU time than most approaches for the cogeneration system. Table 1 reveals that the HDE-MU is a satisfactory algorithm for solving the CHPED problem. The convergence characteristics of the proposed method in comparison with DE with MU (DE-MU) for this real example are depicted in Fig. 5, and the distribution of the minimum costs after 100 trials by the HDE-MU is plotted 
TABLE 2: COMPUTATIONAL RESULTS OF THE DE-MU AND THE PROPOSED HDE-MU FOR THE LARGE SCALE CHPED PROBLEM.

\begin{tabular}{|c|c|c|c|c|c|c|c|c|c|c|}
\hline Methods & \multicolumn{5}{|c|}{ DE-MU, $\left(N_{p}=30\right)$} & \multicolumn{5}{|c|}{ HDE-MU, $\left(N_{p}=10\right)$} \\
\hline No. of units & 20 & 50 & 100 & 200 & 400 & 20 & 50 & 100 & 200 & 400 \\
\hline Cost_av (\$/h) & $54,732.14$ & 136,837 & 274,159 & 555,212 & $1,294,645$ & $54,692.05$ & 136,732 & 273,481 & 547,121 & $1,095,934$ \\
\hline CPU_av (sec) & 17.85 & 42.63 & 88.42 & 193.93 & 435.71 & 5.74 & 14.36 & 28.95 & 58.17 & 122.28 \\
\hline
\end{tabular}

$$
\# \Delta \text { Cost_av }(\%)=\frac{\text { Cost_av of the DE - MU }- \text { Cost_av of the proposed HDE - MU }}{\text { Cost_av of the proposed HDE }-\mathrm{MU}} \text { 100\% }
$$

in Fig. 6. It can be observed that the worst, average and best costs are very close. The small standard deviation again confirms the stability of the proposed HDE-MU.

\section{B. The large scale case}

This test shows that the proposed approach is also applicable to the large scale system of the actual CHPED problem. The basic case given above is expanded to build five systems with 20, 50, 100, 200 and 400 units. To avoid confusion owing to the stochastic nature of the solution methods, computational results of 50 runs beginning with random initial populations were averaged, and are given in Table 2. This table reveals that the proposed approach is superior to the DE-MU in terms of average cost (Cost_av) and average CPU time (CPU_av) in all cases of units. The advantage of the proposed algorithm is mostly in the solution quality and calculation speed, and its superiority becomes clearer as the system size is enlarged. Moreover, the proposed method requires a smaller population size than the DE-MU. Significantly, the computational burden of the proposed algorithm grows almost linearly with respect to the increase in system size, showing that proposed approach is applicable to large scale CHPED problems of power systems.

\section{CONCLUSIONS}

The HDE-MU for solving the CHPED problem has been proposed herein. Complication of the CHPED problem lies in the constraints imposed by the mutual dependencies of multi-demand and Heat-Power capacity. The HDE helps the proposed method efficiently search and refined exploit. The MU helps the proposed method avoid deforming the ALF and resulting in difficulty of solution searching. The proposed algorithm integrates the HDE and the MU that has the merits of taking a wide range of penalty parameters and a small population. A practical CHPED system is used to compare the proposed HDE-MU with fifteen reported methods. Simulation results demonstrate that the proposed algorithm has more advantages for solving the CHPED problem than the previous methods. The contributions of this paper are the MU effectively handles the Heat-Power feasible region constraints, the HDE efficiently searches the optimal solutions in the economic dispatch process, and the author provides a valid and efficacious algorithm for the large scale CHPED problem of power systems.

\section{REFERENCES}

[1] M. Basu, "Group search optimization for combined heat and power economic dispatch," International Journal Electrical Power and Energy Systems, vol. 78, pp. 138-147, 2016.

[2] T. T. Nguyen, D. Ngoc Vo, and B. H. Dinh, "Cuckoo search algorithm for combined heat and power economic dispatch,"
International Journal Electrical Power and Energy Systems, vol. 81, pp. 204-214, 2016.

[3] A. Haghrah, M. Nazari-Heris, and B. Mohammadi-ivatloo, "Solving combined heat and power economic dispatch problem using real coded genetic algorithm with improved Mühlenbein mutation," Applied Thermal Engineering, vol. 99, pp. 465-475, 2016.

[4] A. Meng, P. Mei, H. Yin, X. Peng, and Z. Guo, "Crisscross optimization algorithm for solving combined heat and power economic dispatch problem," Energy Conversion and Management , vol.105, pp. 1303-1317, 2015.

[5] P. K. Roy, C. Paul, and S. Sultana, "Oppositional teaching learning based optimization approach for combined heat and power dispatch," International Journal Electrical Power and Energy Systems, vol. 57, pp. 392-403, 2014.

[6] Q. Niu, H. Zhang, X. Wang, K. Li, G. W. Irwin, "A hybrid harmony search with arithmetic crossover operation for economic dispatch," International Journal Electrical Power and Energy Systems, vol. 62, pp. 237-257, 2014.

[7] M. T. Hagh, S. Teimourzadeh, M. Alipour, P. Aliasghary, "Improved group search optimization method for solving CHPED in large scale power systems," Energy Conversion and Management, vol. 80, pp. 446-456, 2014.

[8] F. J. Rooijers and R. A. M. van Amerongen, "Static economic dispatch for co-generation systems," IEEE Trans Power Systems, vol. 9, no. 3, pp. 1392-1398, 1994.

[9] T. Guo, M. I. Henwood, and M. van Ooijen, "An algorithm for combined heat and power economic dispatch," IEEE Trans. Power Systems, vol. 11, no. 4, pp.1778-1784, 1996.

[10] M. S. Javadi, N. A. Esmaeel, and S. Sabramooz, "Economic heat and power dispatch in modern power system harmony search algorithm versus analytical solution,” Scientia Iranica, vol. 19, no. 6, pp.18201828, 2012.

[11] H. C. Chang and P. C. Lin, "A demonstration of the improved efficiency of the canonical coordinates method using nonlinear combined heat and power economic dispatch problems," Engineering Optimization, vol. 26, no. 2, pp.261-269, 2014.

[12] S. H. Huang and P. C. Lin, "A harmony-genetic based heuristic approach toward economic dispatching combined heat and power," International Journal Electrical Power and Energy Systems, vol. 53, pp.482-487, 2013.

[13] E. Khorram and M. Jaberipour, "Harmony search algorithm for solving combined heat and power economic dispatch problems," Energy Convers and Management, vol. 52, pp. 1550-1554, 2011.

[14] M. A. Mellal and E. J. Williams, "Cuckoo optimization algorithm with penalty function for combined heat and power economic dispatch problem," Energy, vol. 93, pp. 1711-1718, 2015.

[15] S. D. Beigvand, H. Abdi, and M. La Scala, "Combined heat and power economic dispatch problem using gravitational search algorithm," Electric Power Systems Research, vol. 133, pp. 160-172, 2016.

[16] N. Ghorbani, "Combined heat and power economic dispatch using exchange market algorithm," International Journal Electrical Power and Energy Systems, vol. 82, pp. 58-66, 2016.

[17] H. R. Abdolmohammadi and A. Kazemi, "A Benders decomposition approach for a combined heat and power economic dispatch," Energy Convers and Management, vol.71, pp.21-31, 2013.

[18] B. Mohammadi-Ivatloo, M. Moradi-Dalvand, and A. Rabiee, "Combined heat and power economic dispatch problem solution using particle swarm optimization with time varying acceleration coefficients," Electric Power Systems Research, vol. 95, pp. 9-18, 2013.

[19] A. Yazdani, T. Jayabarathi, V. Ramesh, and T. Raghunathan, "Combined heat and power economic dispatch problem using firefly algorithm,” Front Energy, vol.7, no. 2, pp.133-139, 2013.

[20] T. Jayabarathi, A. Yazdani, V. Ramesh, and T. Raghunathan, "Combined heat and power economic dispatch problem using the invasive weed optimization algorithm," Front Energy, vol. 8, no. 1, pp.25-30, 2014. 
[21] A. Vasebi, M. Fesanghary, and S. M. T. Bathaee, "Combined heat and power economic dispatch by harmony search algorithm," International Journal Electrical Power and Energy Systems, vol. 29, pp. 713-719, 2007.

[22] R. Storn and K. V. Price, "Minimizing the real functions of the ICEC 1996 contest by differential evolution,” Proc. 1996 IEEE International Conf. Evolutionary Computation, ICEC'96, pp. 842844, 1996.

[23] C. L. Chiang, "Hybrid Differential Evolution for Power Economic Dispatch Problems Considering the incorporated cost Model," International Journal Applied Mechanics and Materials, vol.302, pp. 782- 786, 2013.

[24] C. L. Chiang, "Power Economic Dispatch Problems Considering Prohibited Operating Zones and Spinning Reserve: a Hybrid Differential Evolution,” International Journal Applied Mechanics and Materials, Vol.229-231, pp. 2701-2707, 2012.

[25] C. L. Chiang, "Hybrid Differential Evolution based Multi-objective Approach for Hydrothermal Power Systems," Int. J. Applied Mechanics and Materials, Vol. 212-213, pp. 1009- 1014, 2012.

[26] C. L. Chiang and C. A. Wang "Improved Differential Evolution for Hydrothermal Power Systems,” Proc. of the 6th IEEE Conference on Industrial Electronics and Applications (ICIEA2011), Beijing, China, June 21-23, pp. 2420-2425, 2011.

[27] C. L. Chiang and C. A. Wang "Hybrid Differential Evolution for Cogeneration Economic Dispatch Problem," Proc. of 2010 International Conference on Machine Learning and Cybernetics (ICMLC 2010), Qingdao, Shandong, China, 11-14 July 2010, IEEE Catalog NO. CFP10523-PRT, vol. 3, pp. 1560-1565.
[28] C. L. Chiang, "Improved genetic algorithm for power economic dispatch of units with valve-point effects and multiple fuels," IEEE Trans. on Power Systems, vol. 20, no. 4, pp. 1690-1699, 2005.

[29] Z. Michalewicz and M. Schoenauer, "Evolutionary algorithms for constrained parameter optimization problems," Evolutionary Computation, vol. 4, no. 1, pp.1-32, 1996.

[30] M. J. D. Powell, “Algorithms for nonlinear constraints that use Lagrangian function,” Math. Programming, vol. 14, pp 224-248, 1978.

[31] C. L. Chiang, C. T. Su, and F. S. Wang, “Augmented Lagrangian method for evolutionary optimization of mixed-integer nonlinear constrained problems," International Mathematics Journal, vol.2, no. 2, pp. 119-154, 2002.

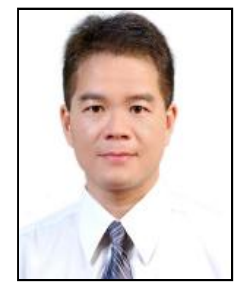

C. L. Chiang received his M.S. degree from Automatic Control Engineering, Feng Chia University, Taichung, Taiwan in 1991, and Ph. D. degree from Institute of Electrical Engineering, National Chung Cheng University, Chia-Yi, Taiwan in 2004.

He is now a professor of Nan Kai University of Technology, Nan-Tou, Taiwan, ROC. His research interests are in the control theory, applications of the optimization, evolutionary algorithms and power economic dispatch. 